\title{
Identification of clays and Fe oxide minerals rich alteration zones using a Landsat 8 image of Pu Sam Cap area, Lai Chau
}

\author{
Hieu Trung Tran $1^{*}$, Cuong Quoc Tran 1, Dung My Tran ${ }^{2}$, Chung Minh Bui ${ }^{3}$, Dung \\ Van Chu ${ }^{2}$, Thanh Trung Nguyen ${ }^{1}$, Quan Cong Nguyen ${ }^{1}$, Anh Duc Nguyen ${ }^{1}$, Thao \\ Phuong Bui ${ }^{1}$ \\ ${ }^{1}$ Institute of Geological Science - VAST, Hanoi, Vietnam \\ 2 General Department of Geology and Minerals of Vietnam, Hanoi, Vietnam \\ ${ }^{3}$ Intergeo Geological Division, Hanoi, Vietnam
}

\section{ARTICLE INFO}

Article history:

Received $17^{\text {th }}$ Nov. 2020

Revised $28^{\text {th }}$ Feb. 2021

Accepted 30 $0^{\text {th }}$ Mar. 2021

\section{Keywords:}

DCPA,

Hydrothermal alteration,

Landsat,

Pu Sam Cap,

Remote Sensing.

\section{ABSTRACT}

The hydrothermal alteration zones are the important sign for mineral exploration and can be identified by remote sensing images completely, but this is limited due to the effect of vegetable. We address this problem by a method called "Directed Principal Component Analysis" (DPCA) that involves calculating principal components on two input band ratio images. One ratio is a geological discriminant, confused by the presence of vegetation; the second ratio is chosen for its suitability as a vegetation index. DPCA applied on Landsat 8 image in Pu Sam Cap area, Lai Châu characteristied by argilic alteration, sericite alteration, etc., with the typical minerals like kaolinite, illite, etc., and pyrite, chalcopyrite, magnetite; specularite, etc., The results have identified Fe - rich zones in Bai Bang and Nam Tra areas; clay minerals are concentrated mainly in Nam Tra area and along the main faults. The results are also compared with previous research data and fieldtrip data that shows similarity and feasibility. This paper indicated limitation of Landsat image such as spatial resolution, spectral resolution, etc., when applied in the tropical area.

${ }^{*}$ Corresponding author

E - mail: trunghieu95ctb@gmail.com

DOI: 10.46326/JMES.2021.62(2).02 


\section{Tạp chí Khoa học Kỹ thuật Mỏ - Địa chất}

Trang điện tử: http://tapchi.humg.edu.vn

\section{Xác định các đới biến đổi giàu khoáng vật sét và oxit sắt sử dụng ảnh Landsat 8 khu vực Pu Sam Cáp, Lai Châu}

\section{Trần Trung Hiếu 1, ${ }^{*}$, Trần Quốc Cường ${ }^{1}$, Trần Mỹ Dũng ${ }^{2}$, Bùi Minh Chung ${ }^{3}$, Chu Văn Dũng ${ }^{2}$, Nguyễn Trung Thành ${ }^{1}$, Nguyễn Công Quân ${ }^{1}$, Nguyễn Đức Anh ${ }^{1}$, Bùi Phương Thảo ${ }^{1}$}

1 Viện Địa Chất - Viện Hàn lâm Khoa học Công nghệ Việt Nam, Hà Nội, Việt Nam

2 Tổng cuc Đia chất và Khoáng sản Việt Nam, Hà Nội, Việt Nam

${ }^{3}$ Liên đoàn Địa chất Intergeo, Hà Nội, Việt Nam

THÔNG TIN BÀI BÁO T TÓM TẮT

Quá trình:

Nhận bài $17 / 11 / 2020$

Sưa xong 28/02/2021

Chap nhận đăng 30/3/2021

\section{Tù̀ khóa:}

Biến đổi nhiệt dịch,

DCPA,

Landsat,

Pu Sam Cáp,

Viễn thám.
Các đới biến đổi nhiệt dịch là dấu hiệu quan trong cho tìm kiếm các mỏ khoáng và có thể được nhận dạng trên ảnh viễn thám. Tuy nhiên điều này bi hạn chế do ảnh hương của yếu tố thực vật. Phân tích thành phần chính định hướng (DCPA) là một phương pháp giúp cải thiện điều trên bao gồm việc tính toán các thành phần chính trên các ảnh chia kênh. Môt tỷ lê chứa thông tin đối tượng địa chất, tỷ lẹ thư hai làm nổi bật thực vật. Phưong pháp áp dụng thử nghiệm trên ảnh Landsat 8 vùng Pu Sam Cáp, Lai Châu, đặc trưng bởi biến đổi argilic hóa, sericit hoá, epidot hoá,... với khoáng vật điển hình như nhóm kaolin, illit,... và pyrit, chalcopyrit, magnetit; specularit,... Kết quả phân tích tù̀ ảnh viễn thám đã xác định các đói giàu Fe tập trung khu vực Bãi Bằng và Nậm Tra; các khoáng vật sét tập trung chủ yếu khu Nậm Tra và dọc theo đứt gãy chính. Kết quả cũng được đối sánh với dũ liệu nghiên cúu trước đó và kiếm chứng thực địa cho thấy có sự tương đồng và tính khả thi. Bài báo đã chỉ ra giới hạn của ảnh Landsat nhu hạn chế về độ phân giải không gian, độ phân giải phổ,... khi áp dụng ở vùng nhiệt đới ẩm.

(C) 2021 Trường Đại học Mỏ - Địa chất. Tất cả các quyền được bảo đảm.

\section{Mở đầu}

Các mỏ khoáng nguồn gốc nhiệt dịch luôn đi kèm với các đới biến đổi nhiệt dịch do phản ứng với đá vây quanh. Không phải đới biến đổi nào cũng đi kèm các thân quặng và ngược lại, nhưng sự có mặt của các đới biến đổi và sự phân bố

\section{${ }^{*}$ Tác giả liên hệ}

E- mail: trunghieu95ctb@gmail.com DOI: 10.46326/JMES.2021.62(2).02 không gian của chúng là dấu hiệu có giá trị cho việc tìm kiếm các mỏ khoáng.

Các đới biến đổi có thể được nhận dạng trên ảnh viễn thám do chúng có đặc điểm phản xạ phổ khác biệt với các thành phần xung quanh. Tại các vùng hoang mạc hoặc bán hoang mạc yếu tố thực vật ít phát triển, các đới biến đổi xuất lộ rõ ràng nên thuận lợi cho việc sử dụng ảnh viễn thám xác định vị trí phân bố tổ hợp khoáng vật của chúng (Sabin, 1999). Tuy nhiên tại các vùng nhiệt đới, thực vật phát triển, giới hạn đáng kể việc nhận 
dạng đới biến đổi do sự phản xạ phổ tương tự của chúng với các đối tượng quan tâm. Do đó các nghiên cứu ứng dụng dữ liệu viễn thám cho tìm kiếm mỏ khoáng cũng bị giới hạn (Pour và nnk., 2013; Carranza, Hale, 2002).

Một số kỹ thuật tăng cường ảnh giúp làm nổi bật các đối tượng quan tâm như chia kênh (band ratio), kỹ thuật Crosta (Cro'sta, Moore, 1989), phân tích thành phần chính định hướng (Directed Principal Component Analysis - DPCA),... Sabins (1999) sử dụng ảnh Landsat TM nhận dạng đới biến đổi nhiệt dịch bằng các ảnh chia kênh được chiết xuất tự động, xác định được hai tổ hợp khoáng vật bị biến đổi là các khoáng vật $\mathrm{Fe}$ và các khoáng sét cùng với alunit tại vùng mỏ $\mathrm{Cu}-\mathrm{Au}$ Goldfield, Nevada (Hoa Kỳ). Shafaroudi và nnk. (2009) cũng sử dụng phương pháp chia kênh trên ảnh ASTER để xác định các khoáng vật biến đổi nhiệt dịch tại Iran. Tangestani và Moore, (2001) xác định các đới biến đổi Cu porphyr bằng so sánh ba kỹ thuật phân tích thành phần chính (PCA). Crosta và nnk. (2003) xác định các khoáng vật biến đổi trong các mỏ nhiệt dịch nhiệt độ thấp vùng Patagonia, Argentina bằng cách sử dụng kỹ thuật PCA trên ảnh ASTER. Fraser và Green (1987) đề xuất phương pháp nhằm hạn chế tối đa ảnh hưởng thực vật với phản xạ phổ đối tượng (DPCA) bằng phân tích PCA trên hai ảnh chia kênh. Kết quả xác định sự phân bố đới sericit, kaolinit, dickit. Fraser (1991) tiếp tục sử dụng phương pháp này xác định đới chứa hematit, goethit.

\section{Sơ lược về khu vực nghiên cứu và dữ liệu sử dụng}

\subsection{Khu vực nghiên cứu}

Vùng $\mathrm{Pu}$ Sam Cáp nằm phần phía Tây Bắc trũng Tú Lệ, gần điểm ranh giới va chạm giữa ba địa khối xảy ra vào Paleozoi Muộn (Subumasu nằm ở phía tây, mảng nam Trung Hoa nằm ở phía bắc và mảng Đông Dương ở phía nam) (Hình $1 \mathrm{~A}$ ). Vùng điển hình với hoạt động magma kiềm giàu $K$, tuổi Paleogen, liên quan hoạt động tách giãn nội mảng do quá trình va chạm Ấn Độ - Âu Á trong Kainozoi sớm và xảy ra trước dịch trượt trái đứt gãy sông Hồng. Hai tổ hợp thạch học chính: (1) lamproit, absarokit, shonkinit mô tả phức hệ $\mathrm{Pu}$ Sam Cáp; (2) trachyt, syenit mô tả hệ tầng Pu Tra và các thành tạo trầm tích cát, bột kết tuổi Trias (Hình 1B) (Trần Trọng Hòa và nnk., 1999;
Trần Đức Lương và Nguyễn Xuân Bao, 1988).

Quặng hóa chủ yếu: đất hiếm và $\mathrm{Au}$ - Cu nhiệt dịch. Vùng Pu Sam Cáp có triển vọng chứa vàng, đồng như khu Bãi Bằng (nằm giữa xã Khun Há Tam Đường và xã Pu Sam Cáp - Sìn Hồ), Nậm Đích, Nậm Tra, Xa Khoáng (xã Noong Hẻo - Sìn Hồ),... (Hình 1B). Các biến đổi thường gặp nhất là biến đổi argilic hóa, thạch anh hoá, sericit hoá, epidot hoá, chlorit hoá. Chúng đều đặc trưng cho quá trình biến đổi nhiệt dịch nhiệt độ thấp với các khoáng vật điển hình như nhóm kaolin, illit,... Các khoáng vật quặng điển hình trong khu vực như pyrit, chalcopyrit, magnetit; specularit,... phân bố ở dạng xâm tán thưa; còn covelin, geothit thường gặp ở dạng các vết bám trong các mặt nứt nẻ ở phần các thân quặng bị phong hóa dang dở. Như vậy, các đá biến đổi nhiệt dịch tại khu vực đặc trưng bởi hai tổ hợp khoáng vật là các khoáng vật sét - alunit và các khoáng vật chứa Fe.

Vùng nghiên cứu có địa hình núi cao, phân cắt mạnh, độ cao dao động $1500 \div 2300 \mathrm{~m}$. Thảm thực vật thưa thớt chủ yếu là rùng thưa tái sinh phát triển trên sườn dốc có nhiều cây gỗ nhỏ, cỏ,...

\subsection{Dũ̃ liệu sử dụng}

Dữ liệu sử dụng trong nghiên cứu gồm bản đồ địa chất và khoáng sản tờ Mường Mới tỉ lệ 1/50.000 (Dương Quốc Lập, 2002); Báo cáo thăm dò vàng đa kim khu vực Bãi Bằng; dữ liệu máy đo phổ cầm tay Spectral Evolution PSR2500 giải đoán bằng aiSIRIS (Công ty TPJ, 2014) và ảnh vệ tinh Landsat 8 chụp ngày 09/02/2019, path/row $(128 / 45)$ lúc 3 h29'. Thời tiết tại thời điểm bay chụp (3h30') tại trạm A Pung $\left(22,18^{0} ; 103,56^{\circ}\right)$ có nhiệt độ $\left(23 \div 32^{\circ} \mathrm{C}\right)$, độ ẩm $(89 \%)$, trời ít mây tan tại độ cao $300 \mathrm{~m}$ (freemeteo.vn).

Máy đo phổ cầm tay Spectral Evolution PSR2500 có khoảng bước sóng $350 \div 2500 \mathrm{~nm}$, giúp cải thiện dữ liệu vệ tinh hoặc đo bay, phục vụ nghiên cứu giải đoán địa chất. Quy trình đo bao gồm: lấy mẫu (đá tươi, mẫu phong hoá, mẫu thổ nhưỡng); lấy chuẩn trắng (độ phản xạ 100\%); đo mẫu (tại mỗi vị trí đo 3 lần với các góc đo khác nhau $90^{\circ} ; 60^{\circ} ; 45^{\circ}$ ). Một số lưu ý khi đo: luôn giữ cố định; khoảng thời gian phù hợp để đo từ $7 \div 11$ $h, 14 \div 16 \mathrm{~h}$; không thực hiện đo phổ khi trời nhiều mây, mưa, ánh sáng tán xạ.

\section{Phương pháp nghiên cứu}



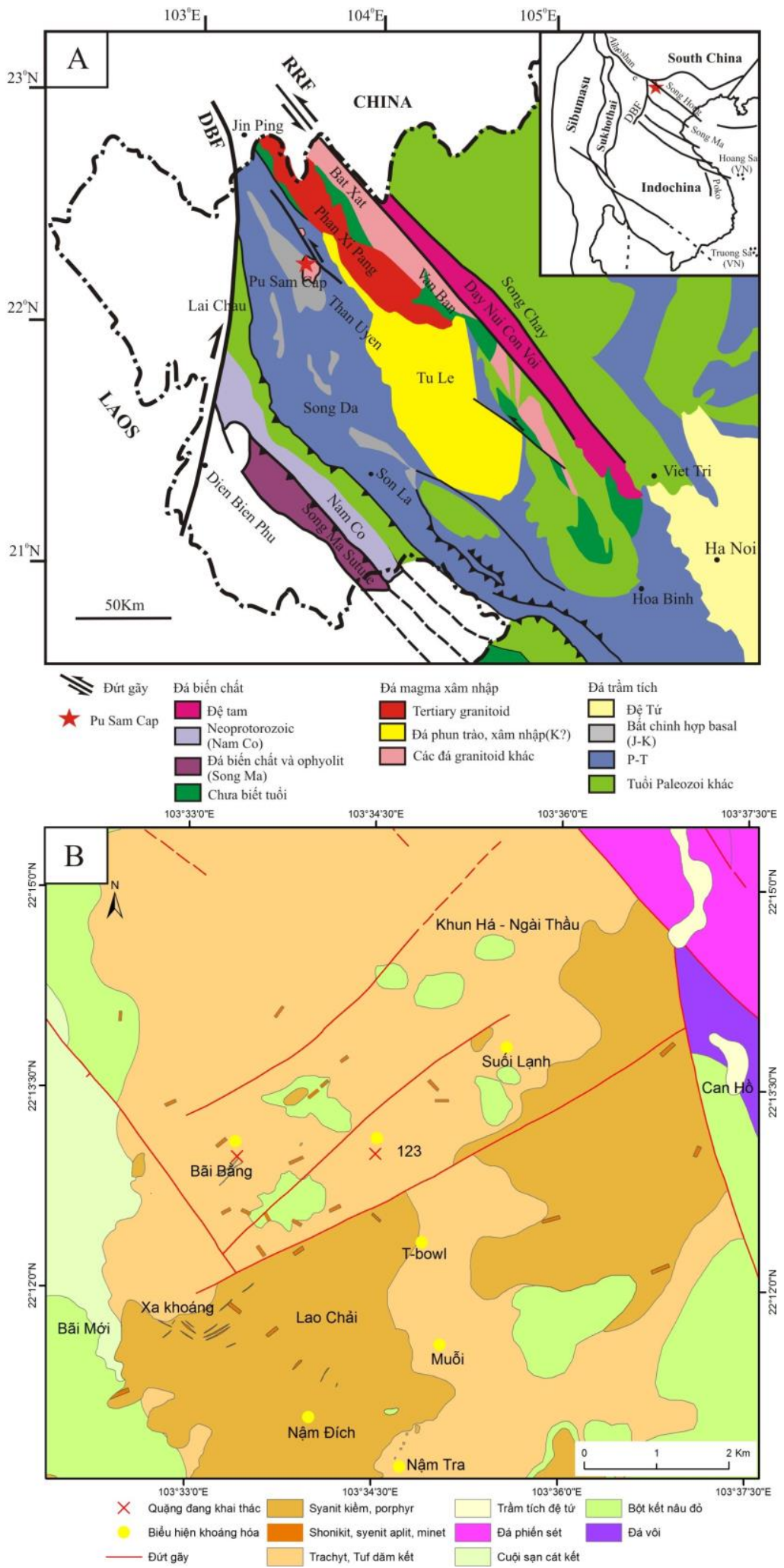

Hình 1. A - Bản đồ giản lược địa chất miền bắc Việt Nam (chỉnh sứa theo Leloup và nnk., 2001); B - Bản đồ địa chất tỷ lệ 1;50.000 vùng Pu Sam Cáp (Dương Quốc Lập, 2002). 


\section{1. Đặc điểm phản xạ phổ một số khoáng vật sét và khoáng vật chứa Fe}

Với khu vực nghiên cứu các đá biến đổi nhiệt dịch đặc trưng gồm các khoáng vật sét - alunit và các khoáng vật chứa Fe nên đây là hai đối tượng nghiên cứu chính. Đặc điểm phản xạ phổ đặc trưng của các khoáng vật là cơ sở quan trọng cho xây dựng các phương pháp phân tích ảnh sau này, giúp xác định sự phân bố, ranh giới của chúng.

Trong vùng hồng ngoại sóng ngắn $(1,1 \div 2,5$ $\mu \mathrm{m})$ các khoáng vật nhóm phyllosilicat, carbonat và sulphat có những phản xạ phổ đặc trưng. Các đá biến đổi sericit, có điểm hấp thụ mạnh, khác biệt liên quan $\mathrm{Al}$ - $\mathrm{OH}$ tại 2,2 $\mu \mathrm{m}$ và một điểm hấp thụ thấp hơn tại 2,35 $\mu \mathrm{m}$ (Spatz, Wilson, 1995). Kaolinit là một khoáng vật điển hình cho biến đổi argillic tiến triển bị hấp thụ mạnh do $\mathrm{Al}$ - $\mathrm{OH}$ tại bước sóng 2,165 $\mu \mathrm{m}$ và 2,2 $\mu \mathrm{m}$ (Hunt, 1977; Hunt, Ashley, 1979)(Hình 2A).

Hematit, goethit, kaolinit, montmorillonit, gibbsit là những khoáng vật phổ biến trong đới vỏ phong hóa nhiệt đới. Trong vùng sóng nhìn thấy và cận hồng ngoại $(0,35 \div 1,1 \mu \mathrm{m})$ các oxit sắt và thực vật bị hấp thụ mạnh ở một số điểm. Các oxit và hydroxit sắt (hematit và goethit) tăng di chuyển $\mathrm{Fe}^{3+}$ khi có sự biến đổi vật lý và hóa học. Hematit có sự hấp thụ mạnh các bước sóng 0,53 ; 0,63 và $0,88 \mu \mathrm{m}$. Goethit bị hấp thụ mạnh tại bước

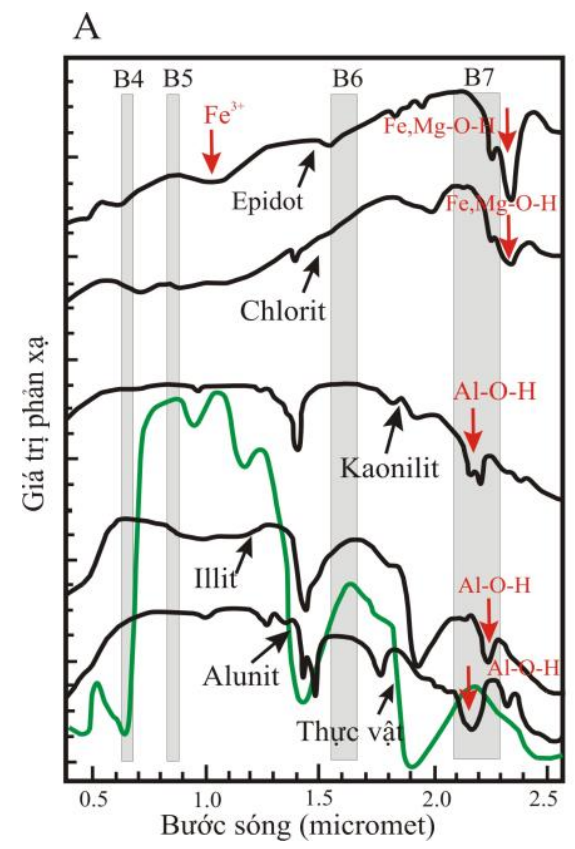

sóng 0,$48 ; 0,67$ và $0,94 \mu \mathrm{m}$ (Hunt và nnk., 1971)(Hình 2B). Sự hấp thụ tại bước sóng 0,54 và $0,48 \mu \mathrm{m}$ của hematit và goethit tương ứng biến đổi kênh đỏ và vàng.

\subsection{Quá trình tiền xử lý dũ̃ liệu}

Dữ liệu ban đầu được hiệu chỉnh bức xạ gồm hai bước chuyển đổi từ các giá trị xám độ sang các giá trị phát xạ và chuyển tiếp qua các giá trị phản xạ. Sau đó dữ liệu tiếp tục được hiệu chỉnh khí quyển nhằm loại bỏ những ảnh hưởng đến phản xạ phổ do yếu tố hơi nước, sương mù,... với thuật toán FLAASH (Fast Line - of - sight Atmospheric Analysis of the Spectral Hypercubes). Thuật toán này áp dụng mô hình chuẩn MODTRAN sử dụng lượng hơi nước dạng cột chuẩn cho mỗi mô hình khí quyển.

\subsection{Phương pháp phân tích thành phần chính định hướng}

Phương pháp phân tích thành phần chính định hướng (DPCA) (Fraser, Green, 1987), tính toán thành phần chính trên các ảnh chia kênh. Ảnh chia kênh bao gồm một tỷ lệ làm nổi bật các đối tượng quan tâm (khoáng vật sét, oxit sắt,...) và một tỷ lệ còn lại làm nổi bật đối tượng gây nhiễu (thực vật,...).

Chia kênh là một phương pháp giúp loại bỏ các ảnh hưởng của chiếu sáng như bóng đổ, góc

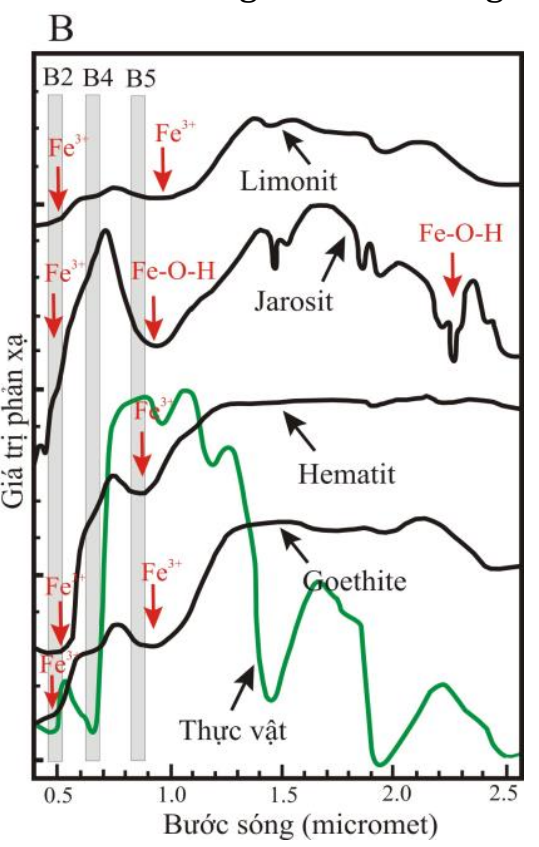

Hình 2. Đồ thị phản xạ phổ một số khoáng vật; thực vật trong phòng thí nghiệm (Clark và nnk., 1993). 
chiếu,... làm nổi bật đối tượng với thành phần xung quanh (Hình 3). Các tỷ lệ được chọn có tử số là kênh có khoảng bước sóng với giá trị phản xạ phổ ít bị biến đổi và mẫu số khoảng bước sóng có giá trị phản xạ phổ biến đổi rõ rệt. Với ảnh Landsat 8, các tỷ lệ thường sử dụng: khoáng vật sét (6/7), thực vật (5/4), khoáng vật chứa oxit sắt (4/2).

Phân tích thành phần chính (PCA) là phương pháp biến đổi tuyến tính các biến sang một hệ tọa độ mới, độc lập tuyến tính và phương sai được sắp xếp chiều giảm dần. Khi đó, có thể xác định các thành phần chính $(\mathrm{PC})$ chứa thông tin quan trọng và loại bỏ các $\mathrm{PC}$ ít quan trọng hơn, thuận lợi trong xử lý và phân tích. Phương pháp sử dụng phân tích thành phần chính không chuẩn hoá tương ứng ma trận hiệp phương sai. Một lưu ý là ảnh trước khi chia kênh, các giá trị phản xạ được đưa về giá trị xám độ dạng 8 bit trong khoảng $0 \div 255$. Sau đó các giá trị tỷ lệ tiếp tục được chuẩn hóa trong khoảng $0 \div 255$ với giá trị trung bình xấp xỉ 128 trước khi tính toán PCA.

Sau khi tính toán PCA, giá trị độ lớn và dấu các hệ số tương quan (loadings) của mỗi kênh tỷ lệ trên thành phần chính sẽ phản ánh đặc điểm của chúng (Johnson, Dean, 2007):

$$
\rho_{(P C i, X k)}=\frac{e_{i k} \cdot \sqrt{\mu_{i}}}{\sqrt{\sigma_{k k}}}
$$

Trong đó: $\rho_{(P C i, X k)}$; $e_{i k}$ - giá trị các hệ số tương quan; vector riêng tương ứng kênh $k, P C i$; $\mu_{i}, \sigma_{k k}$ - giá trị riêng, phương sai kênh $k$ trong ma trận hiệp phương sai.

Các hệ số tương quan có cùng dấu khi có điểm tương tự trên đường cong phản xạ phổ giữa đối

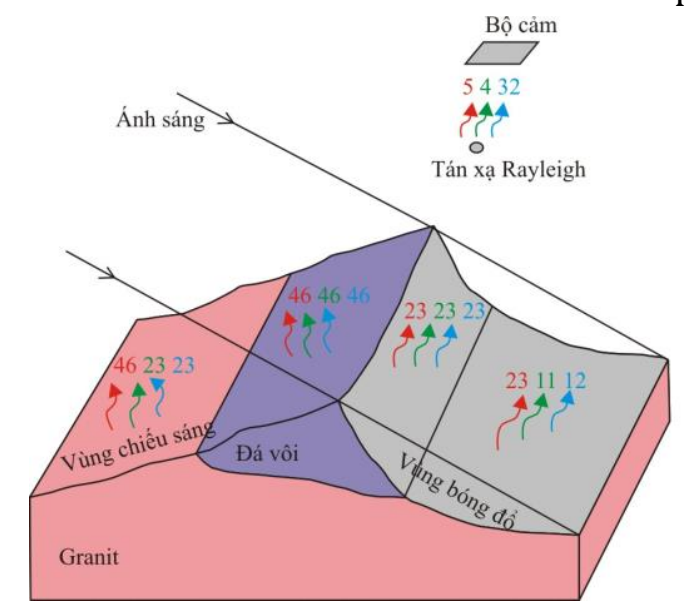

tượng quan tâm và đối tượng gây nhiễu. Nếu các hệ số tương quan có dấu khác nhau, ta sẽ xác định được đối tượng quan tâm thông qua các pixel sáng hay các pixel tối trên ảnh thành phần chính; với hệ số tương quan dương tương ứng pixel sáng và hệ số tương quan âm tương ứng pixel tối (Fraser, Green, 1987). Giá trị độ lớn các hệ số tương quan (giá trị tuyệt đối) phản ảnh mức độ quan trọng của một kênh tỷ lệ trong một thành phần chính và giữa các kênh tỷ lệ với nhau. Kết quả cuối cùng được phân ngưỡng sử dụng dựa trên biểu đồ phân bố các giá trị pixel trên ảnh thành phần chính. Việc phân loại có thể dựa trên kết quả phân tích thống kê, phân chia lớp các giá trị cao theo công thức (De Smith và nnk., 2007):

$$
v=m+2.625^{*} s d
$$

Trong đó: $m$ - giá trị trung bình; $s d$ - giá trị độ lệch chuẩn. Ngoài ra còn có thể phân ngưỡng giá trị cao dựa trên những hiểu biết về đặc điểm địa chất khu vực.

\section{Kết quả và thảo luận}

\subsection{Kết quả}

Với các khoáng vật sét: PC1 chiếm $85.47 \%$ tổng giá trị riêng (Bảng 1 ), chứa phần lớn các thông tin phổ trong vùng. Tất cả các giá trị hệ số tương quan trên PC1 đều âm do đó không thể dùng ảnh PC1 để thể hiện sự khác biệt phổ giữa các đối tượng. Các hệ số tương quan PC2 có sự khác biệt giữa kênh tỷ lệ $6 / 7$ và $5 / 4$. Nhóm khoáng vật sét khi đó thể hiện ở các pixel tối $(-0,392)$, yếu tố thực vật phân bố ở các pixel sáng $(0,313)$. Ngoài ra do độ lớn các hệ số tương quan xấp xỉ nhau nên

Hình 3. Hình mô tả tỷ lệ kênh trước và sau khi hiệu chỉnh khí quyển không phụ thuộc góc chiếu sáng. 
lượng thông tin các kênh tỷ lệ trên các PC2 là gần như nhau.

Với các khoáng vật chứa oxit sắt có thể xác định trên ảnh PC2 ở các pixel tối $(-0,779)$ và thực vật phân bố ở các pixel sáng $(-0,145)$. Ngoài ra, do $0,779>0,145$ nên lượng thông tin ảnh PC2 chiếm phần lớn là các khoáng vật chứa Fe. Đồ thị mô tả (Hình 4) trực quan về sự phân bố các vecto riêng và xu hướng các vecto Pci, chỉ mối quan hệ giữa các đối tượng.

\subsection{Thảo luận}

Theo Bill Howell và nnk. (2007) kiểu tạo khoáng chiếm chủ yếu trong khu vực mang nhiều đặc trưng kiểu mỏ IOCG dựa trên sự biển đổi phổ biến feldspar - hematit ở mức độ cao. Trong đó, specularit chiếm chủ đạo trong đới tạo khoáng và xuất hiện nhiều biến đổi kiểu $\mathrm{Na}$ - Ca hơn so với kiểu mỏ kiềm nhiệt dịch nhiệt độ thấp điển hình.

Kết quả vị trí phân bố các khoáng vật chứa sắt (Hình 5) cho thấy một số điểm tương đồng với thành tạo địa chất khu vực như các đới giàu sắt phần trền khu Bãi Bằng, Muỗi (xã Khun Há - Tam Đường). Chúng phân bố trên hệ tầng Yên Châu với bột, sét kết màu nâu đỏ giàu hàm lượng sắt. Một

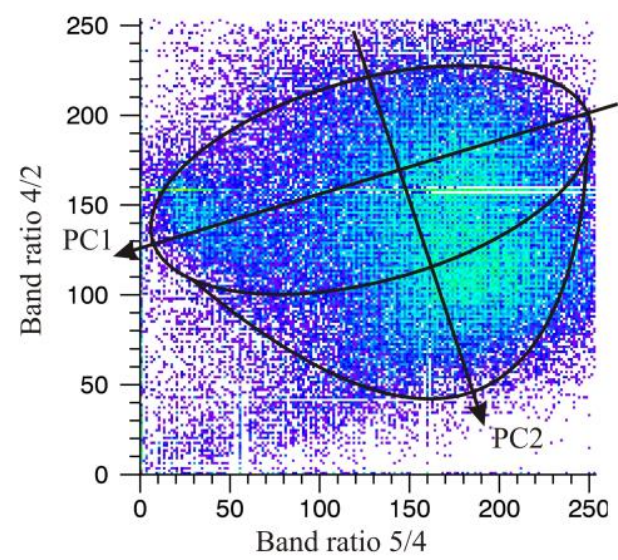

số điểm quặng đang khai thác cũng như điểm có biểu hiện quặng hóa nằm khá gần hoặc nằm trong các đới giàu Fe xác định như Bãi Bằng, 123. Nậm Tra, Nậm Đích (xã Noong Hẻo - Sìn Hồ) (Hình 6B, 6C, 6D). Ngoài ra một đặc điểm quan trọng khoáng hóa khu vực là nằm chủ yếu trong các đới biến đổi chạy dọc theo các đứt gãy; có bề dày, độ dốc, phương thay đổi phụ thuộc các đứt gãy trượt bằng chính và khe nứt tách hoặc các đứt gãy nhỏ dạng đứt gãy thuận kéo theo.

Điều này tăng tính triển vọng các đới phương đông bắc - tây nam kéo dài từ Bãi Bằng đến Khun Hà nằm giữa hai đứt gãy đã xác định, cũng như các đới dạng tuyến khu Nậm Tra, Muỗi.

Đối sánh kết quả với các đới khoáng hóa xác định tại khu vực Bãi Bằng thấy rằng có sự tương đồng; phương đông bắc - tây nam của các đới khoáng hóa trùng với phương các đới khoáng vật chứa Fe đã xác định. Tuy nhiên, sự liên kết này vần chưa rõ ràng do đới khoáng hoá kích thước khá nhỏ 0,5 $\div 2,5$ m (Bùi Minh Chung, 2015) điều này không phù hợp với kích thước pixel ảnh. Đồng thời, do đặc điểm khoáng hoá trong vùng thường đi kèm với magnetit, specularit,... nên đây là một dấu hiệu cho các vùng tiềm năng quặng hoá.

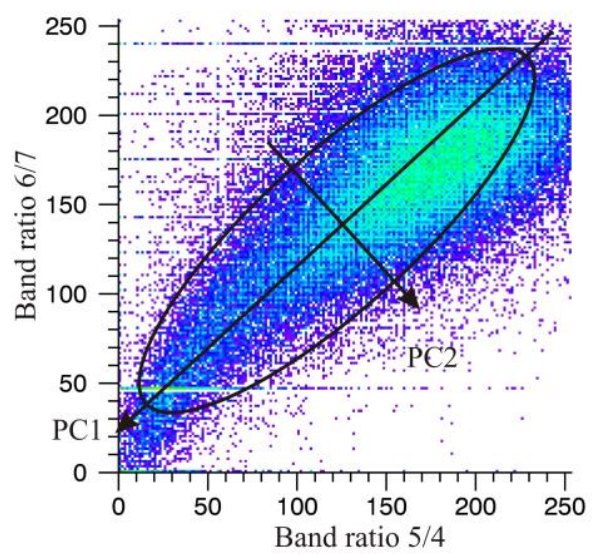

Hình 4. Đồ thị mô tả các vecto riêng.

Bảng 1. Vecto riêng, hệ số tương quan (loadings) trong phân tích thành phần chính các ảnh chia kênh.

\begin{tabular}{|c|c|c|c|c|c|c|c|}
\hline \multirow{2}{*}{$\begin{array}{c}\text { Thành phần } \\
\text { chính (PC) }\end{array}$} & \multicolumn{2}{|c|}{ Vecto riêng } & Giá trị riêng & $\%$ & Phưong sai & \multicolumn{2}{c|}{ Hệ số tương quan } \\
\hline & Kênh tỷ lệ 4/2 & Kênh tỷ lệ 5/4 & & & & Kênh tỷ lệ 4/2 & Kênh tỷ lệ 5/4 \\
\hline PC1 & $-0,183$ & $-0,983$ & 4146,293 & 61,71 & 2624,986 & $-0,230$ & $-1,235$ \\
\hline PC2 & $-0,983$ & 0,183 & 2572,366 & 38,28 & 4093,673 & $-0,779$ & 0,145 \\
\hline & Kênh tỷ lệ 6/7 & Kênh tỷ lệ 5/4 & & & & Kênh tỷ lệ 6/7 & Kênh tỷ lệ 5/4 \\
\hline PC1 & $-0,624$ & $-0,781$ & 6053,202 & 85,47 & 2988,309 & $-0,888$ & $-1,112$ \\
\hline PC2 & $-0,781$ & 0,624 & 1028,780 & 14,52 & 4093,673 & $-0,392$ & 0,313 \\
\hline
\end{tabular}



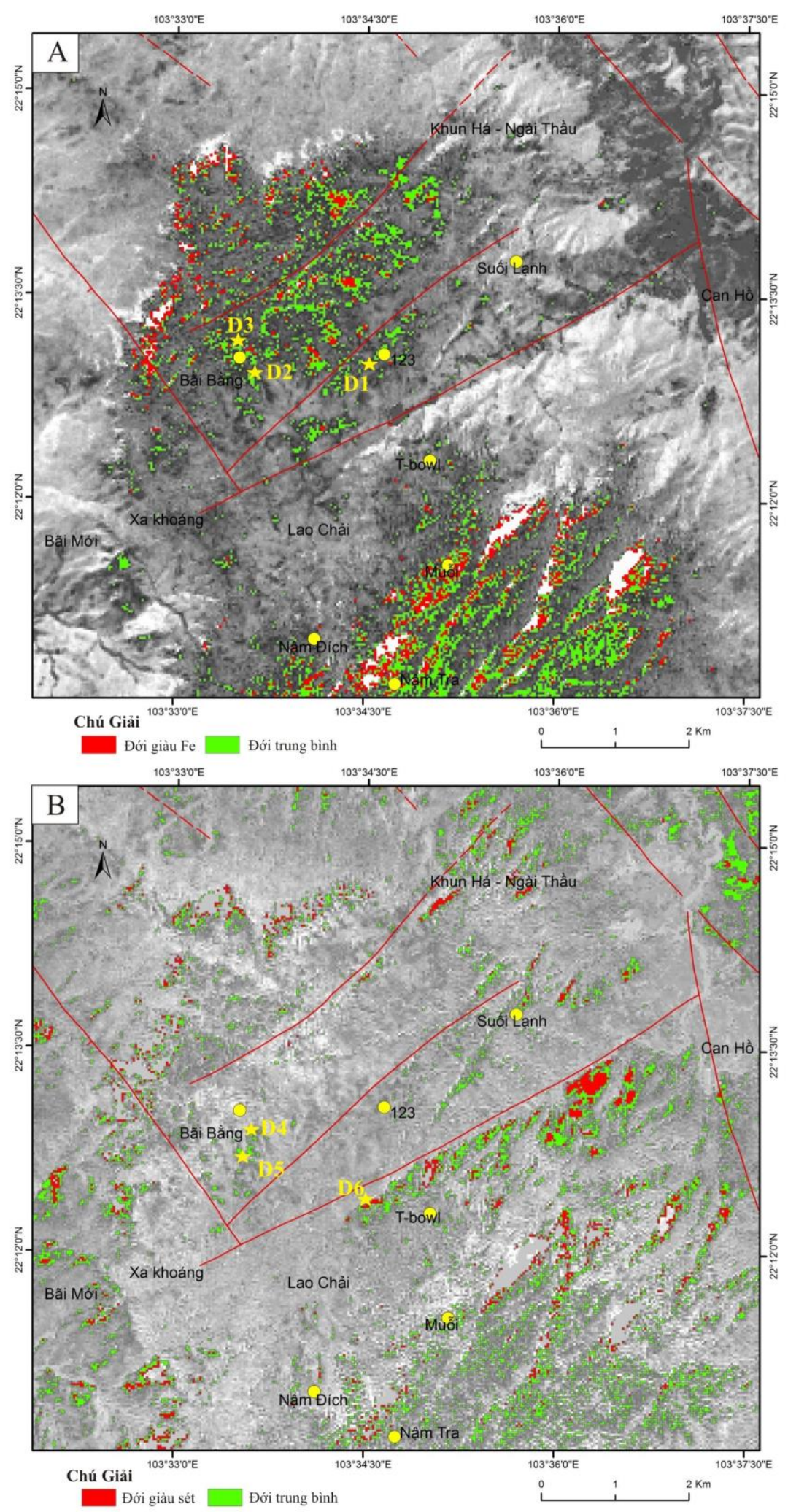

Hình 5. Vị trí các đới giàu khoáng vật.

A - chứa Fe trên ảnh PC2 (4/2 - 5/4); B - khoáng vật sét trên ảnh PC2 (6/7 - 5/4). 

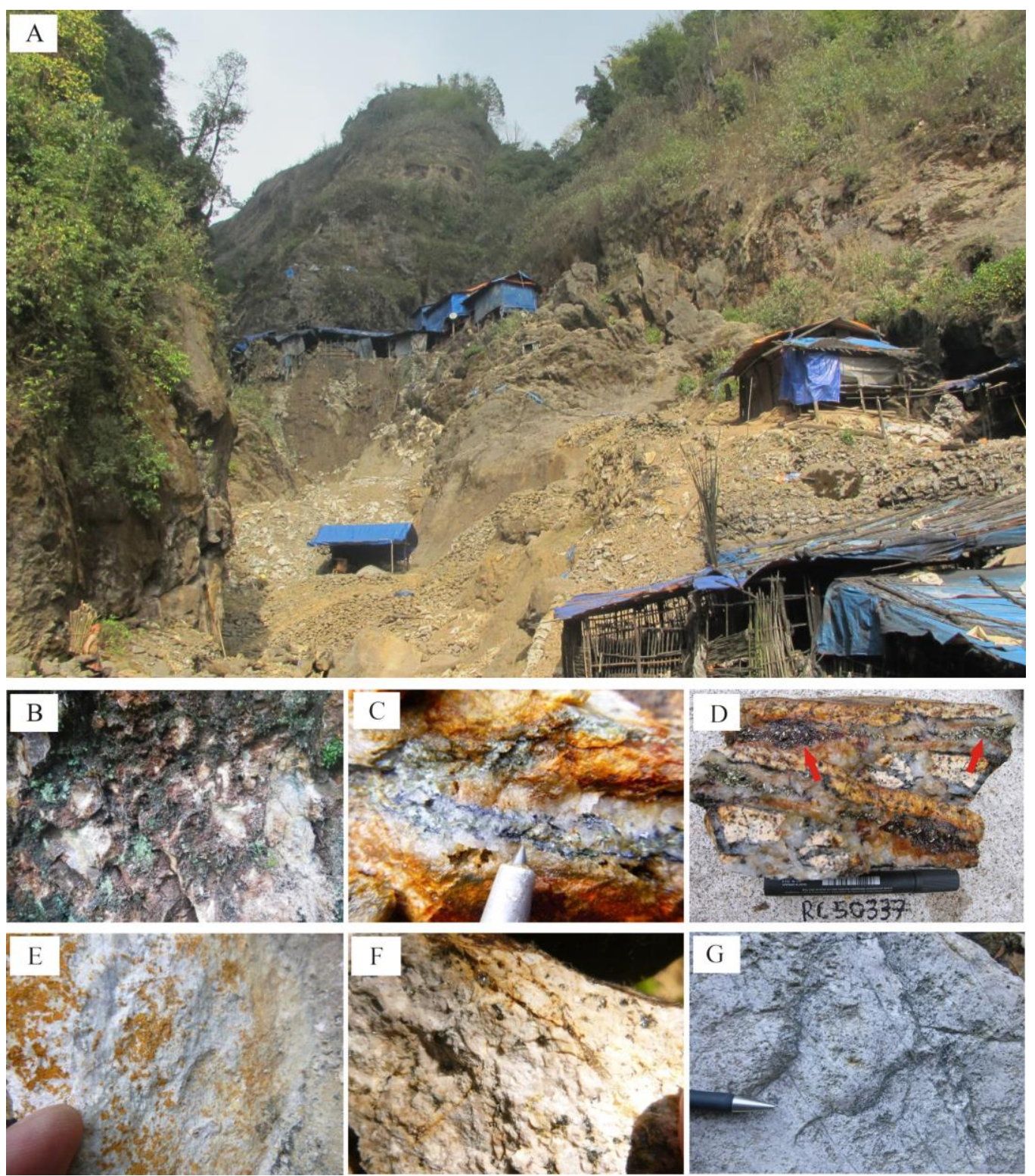

Hình 6. A - Ảnh vị trígần điểm khai thác Bãi Bằng; B - Dăm kết vi trí gần suối Nậm Dịch giàu các hematit - goethit trên bề mặt (Điểm D1, Hình 4A); C - Specularit hình thành trong các mạch thạch anh (Điểm D2, Hình 4A); D - Tinh thể pyrit trong syenit bị biến đổi silic - magnetit - chlorit hoá (Điểm D3, Hình 4A); E Biến đổi argillic hoá đi cùng sự oxi hoá sau biến đổi phyllic hoá (Điểm D4, Hình 4A); F - Biến đổi argillic hoá yếu sau biến đổi kali hoá (Điểm D5, Hình 4A); G - Biến đổi phyllic hoá yếu trên syenit porphyr (Điểm D6, Hìn 4A).

Đối sánh kết quả với dữ liệu máy đo phổ cầm tay (Công ty TPJ, 2014) (Bảng 2, Hình 7) cũng cho thấy có sự tương đồng cao. Dữ liệu đo phổ cầm tay được thực hiện trên cả mẫu đất và mẫu đá, sau đó được phân tích giải đoán bằng aiSIRIS. AiSIRIS là một ứng dụng trí tuệ nhân tạo $(\mathrm{AI})$ tính toán các dữ liệu phổ từ máy đo cầm tay thông qua đối sánh với mẫu chuẩn. Từ đó, cho phép nhận dạng, trích xuất đường cong phản xạ phổ đối tượng. Kết quả phân tích aiSIRIS (Bảng 2) thực hiện tại 78 điểm lấy mẫu đã có nhiều điểm (32/78) trùng kết quả phân tích trên ảnh với thành phần chủ yếu hematit, jarosit, goethit, siderit.

Kết quả các đới khoáng vật sét (Hình 5) cũng cho thấy sự khá tương đồng với thành phần địa chất. Các điểm tập trung cao khu Bãi Mới, Muỗi, 


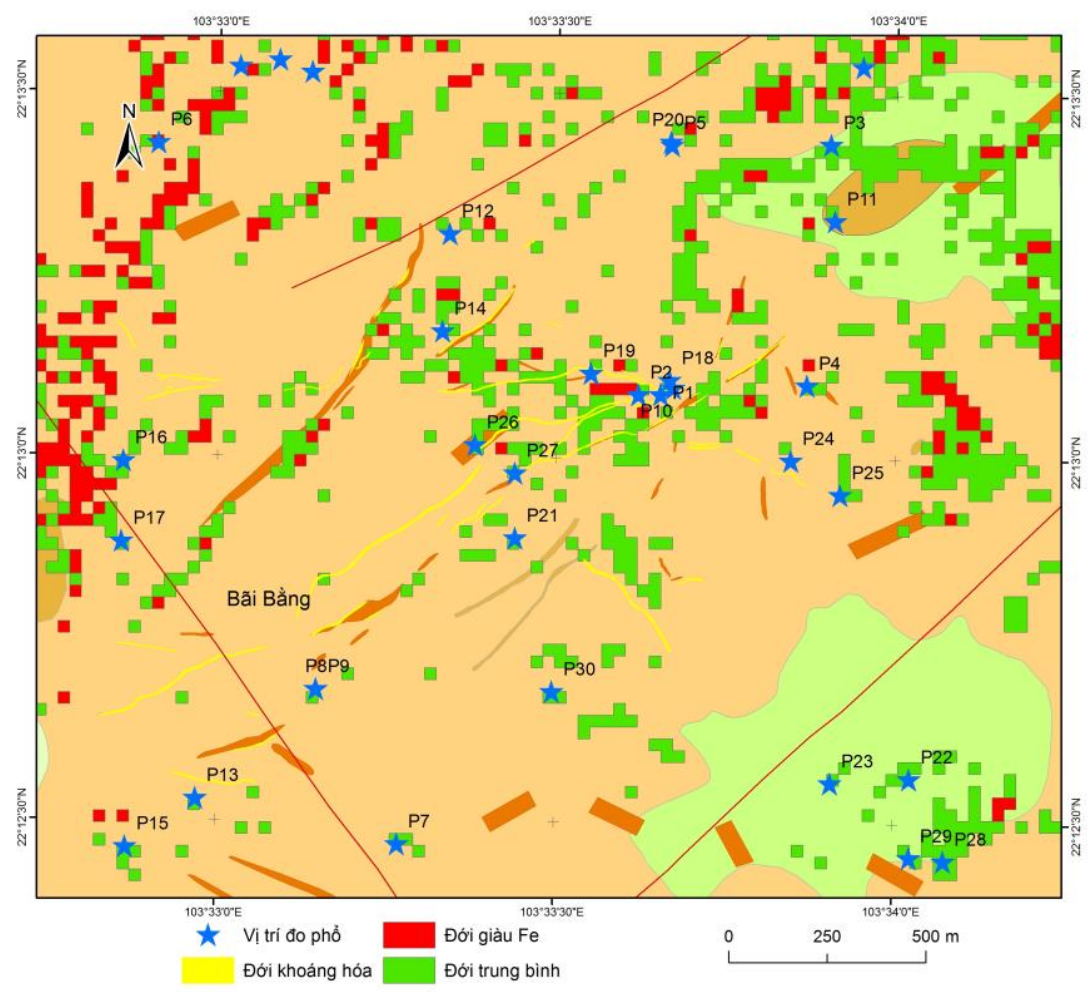

Hình 7. Vị trí thân quặng và điểm đo phổ cầm tay khu Bãi Bằng (Công ty TPJ, 2014).

Bảng 2. Kết quả phân tích máy đo phổ cầm tay (Công ty TPJ, 2014).

\begin{tabular}{|c|c|c|c|c|}
\hline ID & Easting & Northing & VNIRMineral & SWIRMineral \\
\hline 1 & 352198 & 2457702 & goethite+hematite & montmorillonite:95+kaolinite:5 \\
\hline 2 & 352100 & 2457705 & fe carbonate & kaolinite:80+dickite:20 \\
\hline 3 & 352100 & 2457705 & fe carbonate & carbonate \\
\hline 4 & 351674 & 2457675 & hematite+jarosite & gypsum:45+jarosite:30+kaolinite:25 \\
\hline 5 & 351618 & 2457675 & goethite+hematite & gypsum:75+kaolinite:25 \\
\hline 6 & 351889 & 2458319 & goethite+hematite & amphibole:50+water_silica:30+kaolinite:20 \\
\hline 7 & 352108 & 2458307 & goethite & montmorillonite:50+nontronite:45+kaolinite:5 \\
\hline$\ldots$ & $\ldots$ & $\ldots$ & $\ldots$ & $\ldots$ \\
\hline 75 & 351610 & 2456857 & hematite & kaolinite:60+water_silica:40 \\
\hline 76 & 351397 & 2456923 & goethite+hematite & montmorillonite:55+kaolinite:40+white mica:5 \\
\hline 77 & 351761 & 2456846 & hematite & montmorillonite:85+kaolinite:10+chlorite:5 \\
\hline 78 & 351708 & 2456506 & goethite+hematite & montmorillonite:55+gibbsite:25+kaolinite:20 \\
\hline
\end{tabular}

phần trên Can Hồ đều phân bố trên các thành tạo trầm tích cát bột kết, đá phiến sét hệ tầng Yền Châu, Suối Bàng và các thành tạo trầm tích Đệ tứ (Hình 6E, 6F, 6G). Các đới phần trung tâm kéo dài từ $\mathrm{T}$ - bowl đến Can Hồ có cấu trúc dạng tuyến dọc theo đứt gãy chính đông bắc - tây nam. Đồng thời là minh chứng khẳng định sự tồn tại đứt gãy này.

Dữ liệu Landsat khá phổ biến, có nhiều ứng dụng tuy nhiên khi sử dụng nghiên cứu phản xạ phổ đối tượng, vẫn tồn tại một số vấn đề gặp phải như: độ phân giải phổ, độ phân giải không gian. Một số khoáng vật như các khoáng vật sét (kaolinit, illit),... có đường cong phản xạ phổ khá tương tự nhau, thường bị hấp thu mạnh gần bước sóng $2,2 \mu \mathrm{m}$ và chỉ khác biệt một chút với từng khoáng vật. Trong khi độ rộng các kênh trên ảnh Landsat khá lớn nên trên ảnh Landsat rất khó phân biệt được riêng rẽ các khoáng vật sét. Carranza và Hale (2002) giải quyết vấn đề này bằng các tỷ lệ kênh mới dùng cho DPCA (Bảng 3) 
được xây dựng cho mỗi khoáng vật riêng dựa trên đường cong phản xạ phổ của chúng. Tuy nhiên độ chính xác vẫn chưa cao do vẫn tồn tại nhiều ảnh hưởng thực vật.

Bảng 3. Các tỷ lệ kênh sử dụng cho DPCA trên ảnh Landsat 8 (Carranza và Hale, 2002).

\begin{tabular}{|c|c|c|}
\hline \multirow{2}{*}{ Tên } & \multicolumn{2}{|c|}{ Tỷ lệ } \\
\cline { 2 - 3 } & Thực vật & Khoáng vật \\
\hline Quartz & $3 / 4$ & $7 / 2$ \\
\hline Alunit & $5 / 2$ & $6 / 7$ \\
\hline Kaolinit & $5 / 4$ & $6 / 7$ \\
\hline Chlorit & $5 / 3$ & $6 / 2$ \\
\hline Epidot & $3 / 4$ & $6 / 2$ \\
\hline
\end{tabular}

Độ phân giải không gian của các kênh ảnh Landsat phần lớn là 30x30 nên trong khoảng 900 $\mathrm{m}^{2}$ với vùng địa chất phức tạp sẽ chứa rất nhiều thành phần khoáng vật. Như vậy giá trị phản xạ của một pixel là hỗn hợp của nhiều đối tượng, gọi đó là pixel hỗn hợp. Một số chương trình phân loại tự động hiện nay có thể chiết tách phổ các đối tượng trong mỗi pixel hỗn hợp.

Hai vấn đề này của Landsat có thể cải thiện khi sử dụng các ảnh viễn thám khác như ASTER, Hyperion, đặc biệt với dữ liệu siêu phổ PRISMA (Ý) đã cung cấp dữ liệu ảnh từ tháng $5 / 2020, \ldots$ với độ phân giải không gian, độ phân giải phổ tốt hơn.

Một vấn đề khác ảnh hưởng giá trị phản xạ phổ là hiệu chỉnh khí quyển. Bài viết sử dụng thuật toán FLAASH áp dụng mô hình chuẩn MODTRAN, giúp loại bỏ ảnh hưởng các yếu tố như mây, sương mù,... Tuy nhiên, đối khu vực diện tích không quá lớn như khu vực nghiên cứu độ chính xác bị ảnh hưởng. Khi xử lý cần thêm các thông số khí tượng tại thời điểm bay chụp như hàm lượng hơi nước, tỷ lệ $\mathrm{CO}_{2}$, độ cao mây, sương mù,...

Phân tích thành phần chính (PCA) là một phương pháp khá phổ biến trong phân tích ảnh. Có 2 kiểu phân tích thành phần chính là phân tích thành phần chính chuẩn hoá sử dụng ma trận hiệp phương sai và phân tích thành phần chính không chuẩn hoá sử dụng ma trận hệ số tương quan. Khác biệt rõ nhất giữa 2 kiểu trên là sau khi chuẩn hóa các biến, các giá trị riêng sẽ khác nhau dẫn đến phần trăm thông tin trên các PC khác nhau. Các PC đầu trong phân tích thành phần chính chuẩn hoá sẽ có phần trăm thông tin thấp hơn so với trong phân tích thành phần chính không chuẩn hoá. Khi đó các đối tượng không phổ biến trong khu vực sẽ có nhiều thông tin hơn ở các PC sau. Ngoài việc sử dụng các biến đã chuẩn hóa giúp cải thiện đáng kể tỷ lệ tín hiệu/ nhiễu và tăng cường chất lượng ảnh (Singh và Harriso, 1985). Nghiên cứu sử dụng phân tích thành phần chính không chuẩn hoá, tuy nhiên cho kết quả tốt và không sai khác quá nhiều khi sử dụng phân tích thành phần chính chuẩn hoá do lượng kênh thấp.

\section{Kết luận}

Kết quả phân tích chỉ ra một số đới dị thường giàu khoáng vật sét và khoáng vật chứa $\mathrm{Fe}$, từ đó cho phép khái quát về đặc điểm cấu trúc, sự phân bố quặng hóa trong khu vực. Kết quả có sự tương đồng với một số kết quả đối sánh từ dữ liệu giải đoán phổ cầm tay và thực địa. Độ chính xác còn nhiều hạn chế, phụ thuộc nhiều yếu tố như bản chất ảnh, hiệu chỉnh ảnh,... Phương pháp nghiên cứu này sẽ mang lại hiệu quả cao hơn khi có các phương pháp hỗ trợ khác như kết hợp giải đoán ảnh ASTER, Hyperion,...

\section{Lời cảm ơn}

Bài báo hoàn thành là kết quả nghiên cứu từ đề tài cơ sở hỗ trợ cán bộ trẻ năm 2020 của Viện Địa Chất, Viện Hàn lâm Khoa học Công nghệ Việt Nam: "Nghiên cứu đặc điểm phản xạ phổ các khoáng vật sét và khoáng vật chứa sắt sử dụng dữ liệu ảnh Landsat" do tác giả Trần Trung Hiếu làm chủ nhiệm. Tác giả chân thành cám ơn sự hỗ trợ của đề tài "Nghiên cứu xác lập tổ hợp phương pháp điều tra một số loại hình khoáng sản kim loại nội sinh ẩn sâu. áp dụng thí điểm điều tra và khoanh vùng triển vọng vàng gốc ở địa khu Nam Ngãi - Mã số: BĐKH.29/16 - 20" và Công ty TPJ đã hỗ trợ số dữ liệu máy đo phổ cầm tay khu vực nghiên cứu.

\section{Đóng góp của các tác giả}

Trần Trung Hiếu - lên ý tưởng bài báo, viết bản thảo bài báo; Nguyễn Trung Thành, Nguyễn Đức Anh, Bùi Phương Thảo, Nguyễn Công Quân phân tích số liệu; Bùi Minh Chung, Chu Văn Dũng, Trần Quốc Cường, Trần Mỹ Dũng - thu thập dứ liệu, góp ý chỉnh sửa.

\section{Tài liệu tham khảo}

Bill Howell, Nguyễn Thị Thục Anh, Matthew Farmer, Bùi Xuân Vinh. (2007). Some 
preliminary results on the gold exploration program of Pu Sam Cap project of Triple Plate Junction ltd, Viêt Nam. Trung tâm thông tin, lưu trữ và tạp chı địa chat, 9 tr.

Bui Minh Chung. (2015). Đặc điểm quặng hóa vàng gốc khu Bãi Bằng, vùng Pu Sam Cáp, Tam Đường, Lai Châu. Luận văn thạc sl , Trường đại học Mỏ - Địa chất. 101tr.

Carranza, E. J. M., Hale, M. (2002). Mineral mapping with Landsat Thematic Mapper data for hydrothermal alteration mapping in heavily vegetated terrain. Int. J. Remote Sens., 23(22), 4827-4852

Clark, R. N., Swayze, G. A., Gallagher, A., King, T. V. V., Calvin, W. M. (1993). The U.S. geological survey digital spectral library: Version 1: 0.2 to 3.0 um. USGS Open File Report 93 - 592: U.S. Geological Survey, 1340p.

Cong ty TPJ, (2014). Báo cáo thăm dò vàng và đa kim đi kèm vùng Pu Sam Cap, huyện Sìn Hồ và huyện Tam Đường, Lai Châu. Báo cáo nội bộ, Liên đoàn Intergeo và công ty Triple Plate Junction Limited.

Cro'sta, A. P., Moore, J. M. (1989). Enhancement of Landsat Thematic Mapper imagery for residual soil mapping in SW Minas Gerias State, Brazil: a prospecting case history in Greenstone belt terrain. Proceedings of the Seventh Thematic conference on Remote Sensing for Exploration Geology, Calgary, Alberta, Canada, 2 - 6 October 1989 (Ann Arbor, MI: Environmental Research Institute of Michigan). 1173-1187.

Crosta, A. P., C. R. de Souza, F. Azevedo and C. Brodie, (2003). Targeting key alteration minerals in epithermal deposit in Patagonia, Argentina, using ASTER imagery and principal component analysis. Int. J. Remote Sens., $10,4233-4240$.

De Smith, M. J., Goodchild, M. F., Longley, P. (2007). Geospatial Analysis: A Comprehensive Guide to Principles, Techniques and Software Tools. Troubador Publishing Ltd.

Dương Quốc Lập. (cb.). (2002). Bản đồ địa chất và khoáng sản tờ Mường Mới. Trung tâm thông tin, lưu trữ và tạp chı địa chat. Tổng cục địa chất va khoang sản Việt Nam.
Fraser, S. J. (1991). Discrimination and identidentification of Ferric Oxides using satellite Thematic Mapper data: a Newman case study. International Journal of Remote Sensing, 12, 635 - 641.

Fraser, S. J., and Green, A. A., (1987). A software defoliant for geological analysis of band ratios. International Journal of Remote Sensing, 8, 525 $-532$.

Hunt, G., (1977). Spectral signatures of particulate minerals in the visible and near infrared. Geophysics, 42, 501 - 513.

Hunt, G. R., \& Ashley, P. (1979). Spectra of altered rocks in the visible and near infrared.

Econ. Geol., 74, 1613-1629.

Hunt, G. R., Salisbury, J. W., Lenhoff, C. J. (1971). Visible and near - infrared spectra of minerals and rocks: III. Oxides and hydroxides. Mod. Geol., 2, 195-205.

Johnson Richard A., Dean W. Wichern. (2007). Applied Multivariate Statistical Analysis, 6th Edition. Pearson Education, Inc. ISBN 0 - 13 - $187715-1$.

Leloup P. H., Arnaud N., R. Lacassin, J. R. Kienast, T. M. Harrison, T. T. Phan Trong, A. Replumaz, and P. Tapponnier, (2001). New constraints on the structure, thermochronology, and timing of the Ailao Shan - Red River shear Zone, SE Asia. Journal of Geophysical Research, 106(B4), 6683 - 6732.

Pour Amin Beiranvand, Mazlan Hashim, John van Genderen. (2013). Detection of hydrothermal alteration zones in a tropical region using satellite remote sensing data: Bau goldfield, Sarawak, Malaysia. Ore Geology Reviews, 54, 181-196.

Sabins Floyd F., (1999). Remote sensing for mineral exploration. Ore Geology Reviews, 14, 157-183.

Shafaroudi, A. M., M. H. Karimpour, C. R. Stern and S. A. Mazaheri, (2009). Hydrothermal alteration mapping in SW Birjand, Iran, using the Advanced Spaceborne Thermal Emission and Reflection radiometer (ASTER) image processing. J. Applied Sci., 9, 829-842.

Singh, A. and Harrison, A., (1985). Standardized 
Principal Components. International Journal of Remote Sensing, 6(6), 883-896.

Spatz, D. M., Wilson, R. T. (1995). Remote sensing characteristics of porphyry copper systems, western America Cordillera. In: Pierce, F.W., Bolm, J.G. (Eds.), Arizona Geological Society Digest, 20, 94-108.

Tangestani, M. H., and F. Moore, (2001). Comparison of three principal component analysis techniques to porphyry copper alteration mapping: A case study, Meiduk area, Kerman, Iran.Can. J. Remote Sens., 27, 176-182.

Trần Đức Lương, Nguyễn Xuan Bao. (1988). Bản đồ địa chất Việt Nam tỷ lệ 1:500.000. Tổng cục Mỏ và Địa chất. Hà Nội.

Trần Trọng Hòa, Hoàng Hữu Thành, Ngô Thị Phượng, Trần Tuấn Anh, Hoang Việt Hằng. (1999). Các đá magma kiềm kali Tay Bắc Việt Nam: biểu hiện tach gian nội mảng Paleogen muộn. Tạp chí địa chất, $A(7-14)$. 